\title{
Eosinophilic Reaction at the Time of Catheter Insertion Predicts Survival in Patients Initiating Peritoneal Dialysis
}

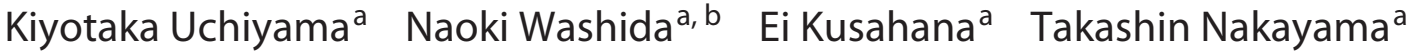 \\ Kohkichi Morimoto ${ }^{\mathrm{a}}$ Hiroshi Itoh $^{\mathrm{a}}$ \\ aDivision of Endocrinology, Metabolism and Nephrology Department of Internal Medicine, Keio University School \\ of Medicine, Tokyo, Japan; bepartment of Nephrology, International University of Health and Welfare School of \\ Medicine, Chiba, Japan
}

\section{Keywords}

Eosinophil · Heart failure - Hypersensitivity - Peritonitis .

Technique survival

\begin{abstract}
Introduction: Despite excellent biocompatibility, insertion of silicone peritoneal dialysis (PD) catheters can induce minor foreign body reaction, affecting long-term patient outcomes. We evaluated the effect of eosinophilic reaction associated with PD catheter insertion on outcomes of patients initiating PD. Methods: Eosinophilic reaction to PD catheter insertion was defined as the ratio of peripheral blood eosinophil count at 1 week after insertion (E1W) to pre-insertion eosinophil count (E0), and the association of E1W/E0 with technique survival, peritonitis-free survival, and heart failure (HF)-related hospitalization-free survival was analyzed. $\boldsymbol{R e}$ sults: This retrospective cohort study included 116 patients (89 male patients) who underwent PD catheter insertion between January 2008 and June 2018 (61.3 \pm 12.9 years). The follow-up duration was $46.2(23.8-75.3)$ months. E1W was significantly higher than E0 (median, 333 vs. $234 / \mu \mathrm{L}, p<$ 0.001 ), with a median E1W/E0 of 1.54. The log-rank test showed that technique survival, peritonitis-free survival, and
\end{abstract}

karger@karger.com www.karger.com/bpu

Karger $\stackrel{\text { ' }}{5}$

GOPEN ACCESS
(C) 2021 The Author(s)

Published by S. Karger AG, Basel

This is an Open Access article licensed under the Creative Commons Attribution-NonCommercial-4.0 International License (CC BY-NC) (http://www.karger.com/Services/OpenAccessLicense), applicable to the online version of the article only. Usage and distribution for commercial purposes requires written permission.
HF-related hospitalization-free survival were significantly better in patients with E1W/EO $<1.54$ than in those with E1W/E0 $\geq 1.54$ ( $p=0.002,<0.001$, and $<0.001$, respectively). By the Cox regression analysis adjusted for age, sex, the Charlson comorbidity index, the estimated glomerular filtration rate, and the geriatric nutritional risk index, E1W/EO remained a significant risk factor for technique failure, peritonitis, and hospitalization for HF (hazard ratio (HR) 1.68, $p=$ 0.01 ; HR 2.19, $p<0.001$; HR 2.15, $p<0.001$, respectively). Conclusion: Eosinophilic reaction at the time of PD catheter insertion is a novel marker that may predict outcomes in patients initiating PD.

(c) 2021 The Author(s).

Published by S. Karger AG, Basel

\section{Introduction}

Continuous improvements in peritoneal dialysis (PD)specific catheters have led to the widely used silicone rubber catheters with polyester cuffs providing improved catheter insertion, lower incidence of catheter migration and fluid leakage, and less irritation to peritoneum accompanied by less protein loss [1]. However, despite high biocompatibility of the currently used catheters, increased 
eosinophil count (EC) in the peripheral blood and peritoneal fluid following foreign body reaction triggered by catheter replacement has been widely reported; this reaction can range from mild elevation in asymptomatic patients to severe elevation, leading to eosinophilic peritonitis [2-9]. Short-term blood and peritoneal eosinophilia have not been shown to cause adverse effects on peritoneal function or increase susceptibility to peritonitis [4, $10,11]$; however, its long-term effects on outcomes in patients on PD have not been reported.

We hypothesized that even a small increase in EC at the time of PD catheter insertion might affect long-term outcomes in patients on $\mathrm{PD}$, presumably through interaction with peritoneal membrane function. Several case reports demonstrated pathological changes in the peritoneal membrane after chronic peritoneal fluid eosinophilia, causing encapsulating peritoneal sclerosis (EPS) in 1 case $[12,13]$. We evaluated the association of eosinophilic reaction with $\mathrm{PD}$ catheter insertion with technique survival as well as peritonitis and heart failure (HF).

\section{Materials and Methods}

\section{Study Population}

This is single-center retrospective cohort study, and all protocols were approved by the Ethics Committee of the study hospital (approval number: 20200139). The study included all patients $>20$ years who were introduced to PD in our hospital between January 1, 2008 and June 30, 2018 and who provided consent for participation. Patients without data on peripheral blood ECs before and/or 1 week after PD catheter insertion were excluded.

\section{Data Collection and Patient Evaluation}

The following demographic data at the time of PD catheter insertion were obtained from medical records: age, sex, PD modality (continuous ambulatory or automated), the method of PD initiation (short-term PD induction and education or stepwise PD initiation using the Moncrief-Popovich technique), diabetes mellitus, history of cerebrocardiovascular disease, type of antihypertensive agents or diuretics used, catheter type, and type and duration of antibiotics used in the perioperative period. The Charlson comorbidity index (CCI) was calculated from the records. The geriatric nutritional risk index (GNRI) was calculated using body weight and serum albumin level [14]. The dialysis/plasma creatinine ratio at $4 \mathrm{~h}(\mathrm{D} / \mathrm{P} 4)$ measured by a standard peritoneal permeability test within 6 months of PD catheter insertion was also obtained from the records.

\section{PD Initiation and Eosinophilic Reaction Evaluation}

As a general practice, catheter insertion was performed under spinal anesthesia. Only silicone rubber swan-neck catheters with double or triple polyester cuffs were used in this study [1]. PD fluid dwelling was initiated 1 week after surgery, during which short-term PD induction and the education method were used. Perioperative treatment with antibiotics, which usually included cefazolin sodium, was initiated before the catheter replacement surgery and continued until postoperative day 5 . We aimed to evaluate eosinophilic reaction to the PD catheter itself and not to antibiotics or PD fluid. A decrease in eosinophil percentage is observed with an increase in neutrophil percentage during the first several days following invasive surgery; thus, we adopted peripheral blood EC on postoperative day 7 before the initiation of the first PD fluid dwelling (E1W) divided by peripheral blood EC before PD catheter insertion (E0) (E1W/E0) as an independent variable to evaluate eosinophilic reaction to PD catheter insertion. Additionally, ECs at 1 and 3 months and at 1 year after PD catheter replacement were determined as reference values. PD induction was performed with a $\mathrm{pH}$-targeted low-glucose degradation product dextrose-based PD fluid (1.5\% Dianeal-N PD-2 or Reguneal HCa, pH 6.5-7.5; Baxter, Tokyo, Japan).

\section{Follow-Up}

Participants were divided into low- and high-E1W/E0 groups according to median E1W/E0 values $(\geq 1.54$ and $<1.54)$. All participants were followed up until PD cessation, death, kidney transplantation, or study completion (June 30, 2020). The primary outcome was technique survival time, that is, length of survival without transition to hemodialysis. Participants who died or received kidney transplant were censored, as recommended by the International Society for Peritoneal Dialysis [15]. Peritonitis-free survival time and HF-related hospitalization-free survival time were analyzed as secondary endpoints because peritonitis and HF are 2 leading causes of PD discontinuation in Japan [16].

\section{Statistical Analysis}

Continuous variables were described as means \pm standard deviation or median (25th-75th percentiles) based on normality assessed by the Shapiro-Wilk test, and binary variables were presented as percentages. Normally and nonnormally distributed continuous variables were evaluated by unpaired Student's $t$ test and the Mann-Whitney $U$ test, respectively, and the $\chi^{2}$ test was used for binary variables to compare parameters between groups. Friedman's test with post hoc analysis using Bonferroni correction was used to compare serial changes in peripheral blood ECs. Survival curves were plotted by the Kaplan-Meier method and compared by the log-rank test. Adjusted Cox proportional hazards models were adopted to determine hazard ratios (HRs) with $95 \%$ confidence intervals for survival. In addition to E1W/E0, parameters that were previously shown to be associated with technique survival were included in the multivariate regression model. Consequently, taking multicollinearity into consideration, age, sex, CCI, eGFR, GNRI, and E1W/E0 were included as candidate-independent variables [17-21]. For sensitivity analyses, E0 or E1W or both was included as independent variables, instead of E1W/E0. The same variables were selected as potential covariates for multivariate analysis to assess their association with peritonitis-free survival and HF-related hospitalization-free survival. Additionally, as death and transplantation were competing risk events against technique failure, the Fine and Gray subdistribution hazards model was used in the multivariate model as a sensitivity analysis for technique survival, together with the standard Cox regression model for cause-specific hazards [21]. All statistical analyses were performed using SPSS software for Macintosh (version 25; IBM, Armonk, NY, USA). A 2-tailed $p$ value $<0.05$ was considered to indicate statistical significance. 
Fig. 1. Flowchart of the study participants.

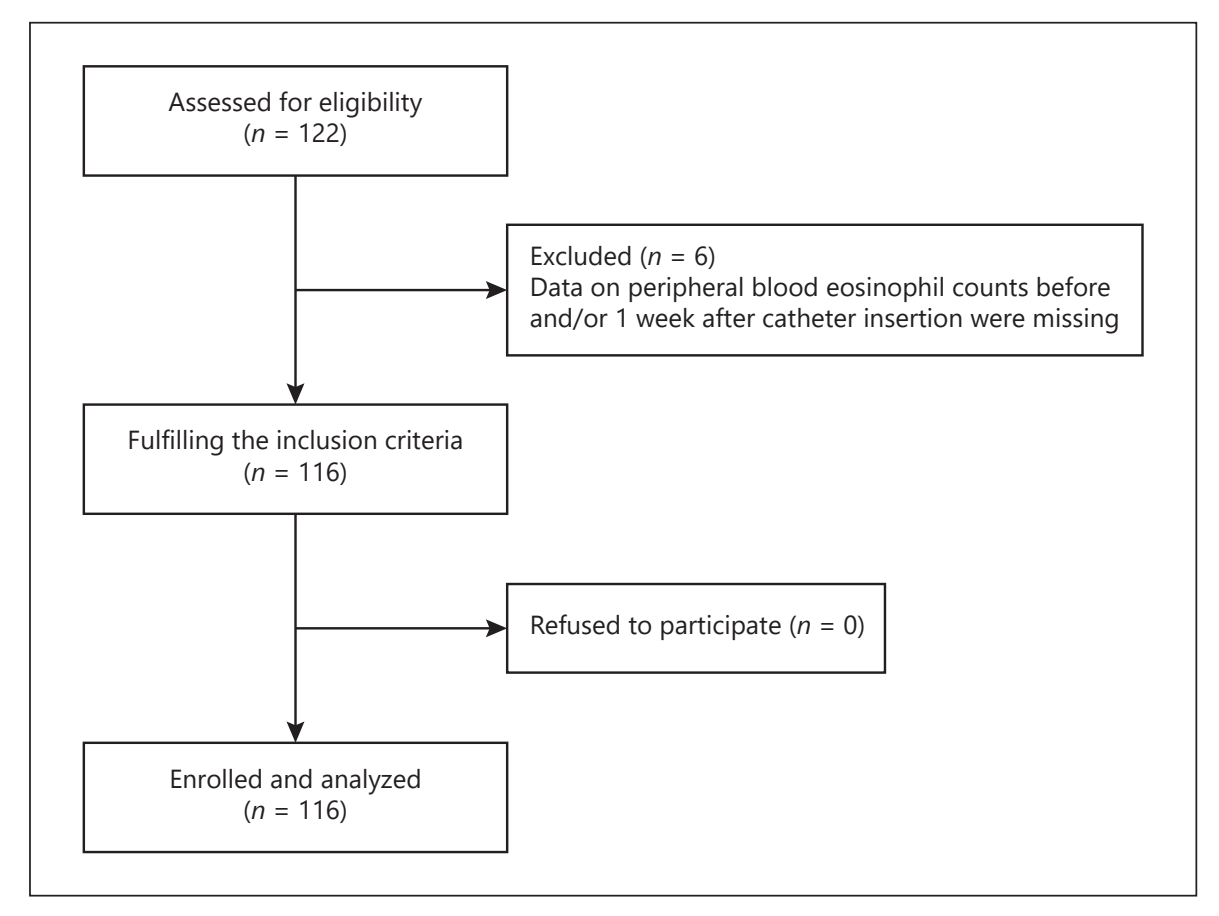

\section{Results}

\section{Patient Clinical Characteristics}

Among 122 eligible patients, 6 patients were excluded because of missing values for E0 and/or E1W; therefore, the study cohort included 116 patients (shown in Fig. 1). Table 1 summarizes the clinical characteristics of the study population and the 2 groups categorized according to median E1W/E0. In the study cohort, the median follow-up period was $46.2(23.8-75.3)$ months, 45 (39\%) patients discontinued PD and were transferred to hemodialysis, $52(45 \%)$ patients developed peritonitis, and 53 (46\%) patients were hospitalized due to HF during the study period. Seven (6\%) patients underwent kidney transplantation, and $24(21 \%)$ patients died without a change in PD modality. There was no loss to follow-up. In contrast, patients who refused participation were predominantly male $(83 \%)$, with a mean age of $56.5 \pm 8.4$ years and a median follow-up period of $54.6 \pm 7.2$ months; these parameters were not significantly different from the study participants.

The high E1W/E0 group ( $n=58)$ exhibited significantly lower eGFR values, lower E0, and higher E1W/E0 compared with the low-E1W/E0 group $(n=58 ; p=0.03$, $<0.001$ and $<0.001$, respectively), whereas the remaining characteristics were not significantly different between the groups. D/P4, obtained at 1-year follow-up, signifi- cantly increased from $0.57 \pm 0.11$ (baseline) to $0.73 \pm 0.14$ in the high-E1W/E0 group $(n=47 ; p<0.001)$ and from $0.59 \pm 0.14$ to $0.65 \pm 0.13$ in the low-E1W/E0 group $(n=$ $48 ; p=0.01$ ). The increase in $\mathrm{D} / \mathrm{P} 4$ was significantly greater in the high E1W/E0 group than in the low-E1W/E0 group ( $0.17 \pm 0.15$ vs. $0.05 \pm 0.14$, respectively; $p<0.001)$. Cefazolin was administered perioperatively in all patients, except for 1 patient in each group treated with vancomycin and 1 patient in the high-E1W/E0 group treated with ampicillin/sulbactam. In the cohort, ECs were significantly higher at 1 week, 1 month, 3 months, and 1 year after PD catheter replacement than at E0 $(p<0.001$ for all), although there was no significant difference (shown in Fig. 2).

\section{Association between Eosinophilic Reaction and Technique Survival}

A total of 27 (47\%) patients in the high-E1W/E0 group and $18(31 \%)$ patients in the low-E1W/E0 group were transferred to hemodialysis. The median technique survival time was significantly lower in the high-E1W/E0 group than in the low-E1W/E0 group (66.4 vs. 104.1 months, respectively; $p=0.002$ ) (shown in Fig. 3a). In addition, the cumulative incidence of technique failure was significantly lower in the high-E1W/E0 group than in the low E1W/E0 group ( $p=0.002$ by Gray's test; Fig. 3b). HD transfer occurred due to peritonitis $(n=16)$, difficulty in 
Table 1. Baseline characteristics of the study population and groups divided according to the median of eosinophilic reaction

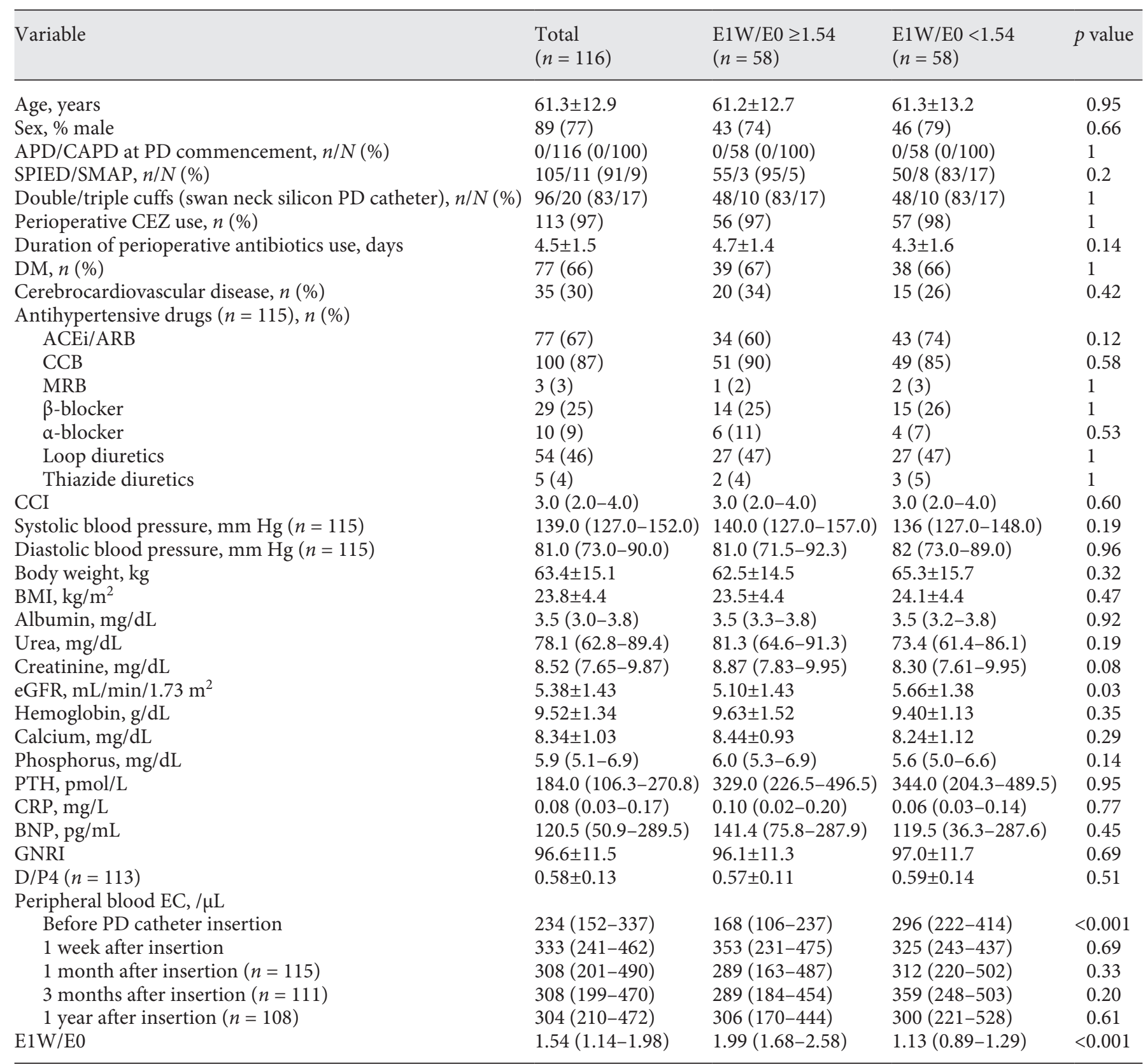

APD, automated peritoneal dialysis; CAPD, continuous ambulatory peritoneal dialysis; PD, peritoneal dialysis; SPIED, short-term PD induction and education; SMAP, stepwise initiation of PD using the Moncrief-Popovich technique; CEZ, cefazolin; ACEi/ARB, angiotensin converting enzyme inhibitor/angiotensin II receptor blocker; CCB, calcium channel blocker; MRB, mineralocorticoid receptor blocker; BMI, body mass index; eGFR, estimated glomerular filtration rate; PTH, parathyroid hormone; CRP, C-reactive protein; BNP, brain natriuretic peptide; GNRI, geriatric nutritional risk index; D/P4, dialysis-to-plasma ratio of creatinine at 4 h; E1W, peripheral blood eosinophil count at 1 week after peritoneal dialysis catheter insertion; E0, peripheral blood eosinophil count before peritoneal dialysis catheter insertion; CCI, Charlson comorbidity index; EC, eosinophil count; DM, diabetes mellitus. 


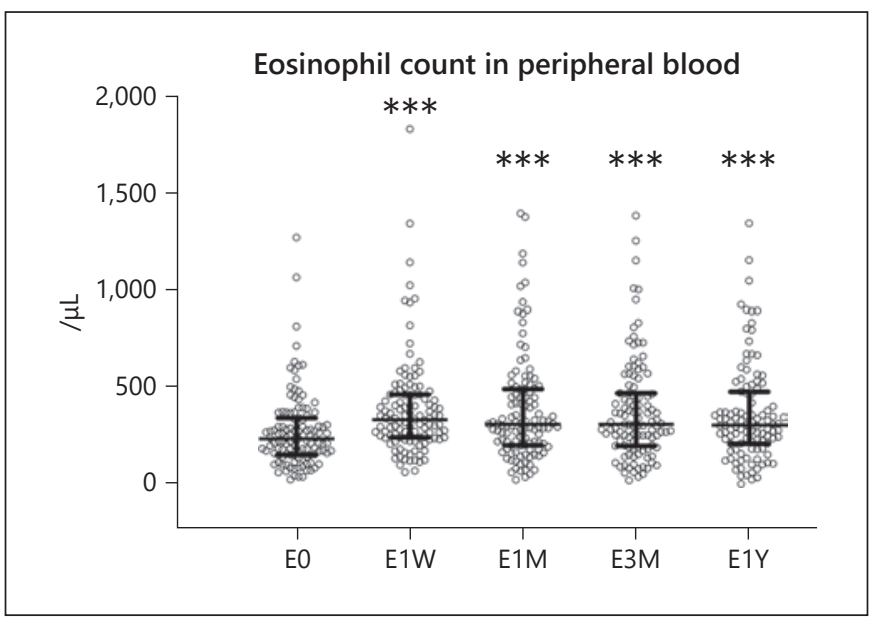

Fig. 2. Serial changes in peripheral EC. Peripheral blood ECs at the time of PD catheter insertion (E0) and at 1 week (E1W), 1 month $(\mathrm{E} 1 \mathrm{M}), 3$ months $(\mathrm{E} 3 \mathrm{M})$, and 1 year $(\mathrm{E} 1 \mathrm{Y})$ after PD catheter replacement. Bars represent medians with an interquartile range. ${ }^{* * *} p<0.001$ versus E0. PD, peritoneal dialysis; ECs, eosinophil counts.

controlling volume overload $(n=4)$, uremic solute retention $(n=2)$, difficulties in performing PD due to decreased activities of daily living $(n=1)$, cerebrovascular disease leading to physical disability $(n=1)$, major abdominal surgery $(n=2)$, and severe eosinophilia $(n=1)$ in the high-E1W/E0 group. In the low-E1W/E0 group, HD transfer occurred due to peritonitis $(n=6)$, difficulty in controlling volume overload $(n=2)$, uremic solute retention $(n=2)$, difficulties in performing PD due to decreased activities of daily living $(n=3)$, cerebrovascular disease leading to physical disability $(n=1)$, catheter malfunction $(n=1)$, major abdominal surgery $(n=1)$, tunnel infection $(n=1)$, and pleuroperitoneal communication $(n=1)$. The rate of peritonitis as a cause of technique failure tended to be higher in the high-E1W/E0 group than in the low-E1W/E0 group $(p=0.13)$.

The standard Cox regression analysis using a causespecific hazard model revealed that higher E1W/E0 and higher CCI were independently associated with technique failure (HR 1.68, $p=0.01$ and $\operatorname{HR} 1.47, p=0.002$, respectively), whereas male sex was associated with a significantly higher risk of technique failure (HR 2.44, $p=$ 0.04 ) (Table 2). This model had a sufficiently high concordance index of $>0.70$. The subdistribution hazard regression model revealed a similar result, wherein higher E1W/E0 and higher CCI were significantly and independently associated with technique failure (HR 1.56, $p=$

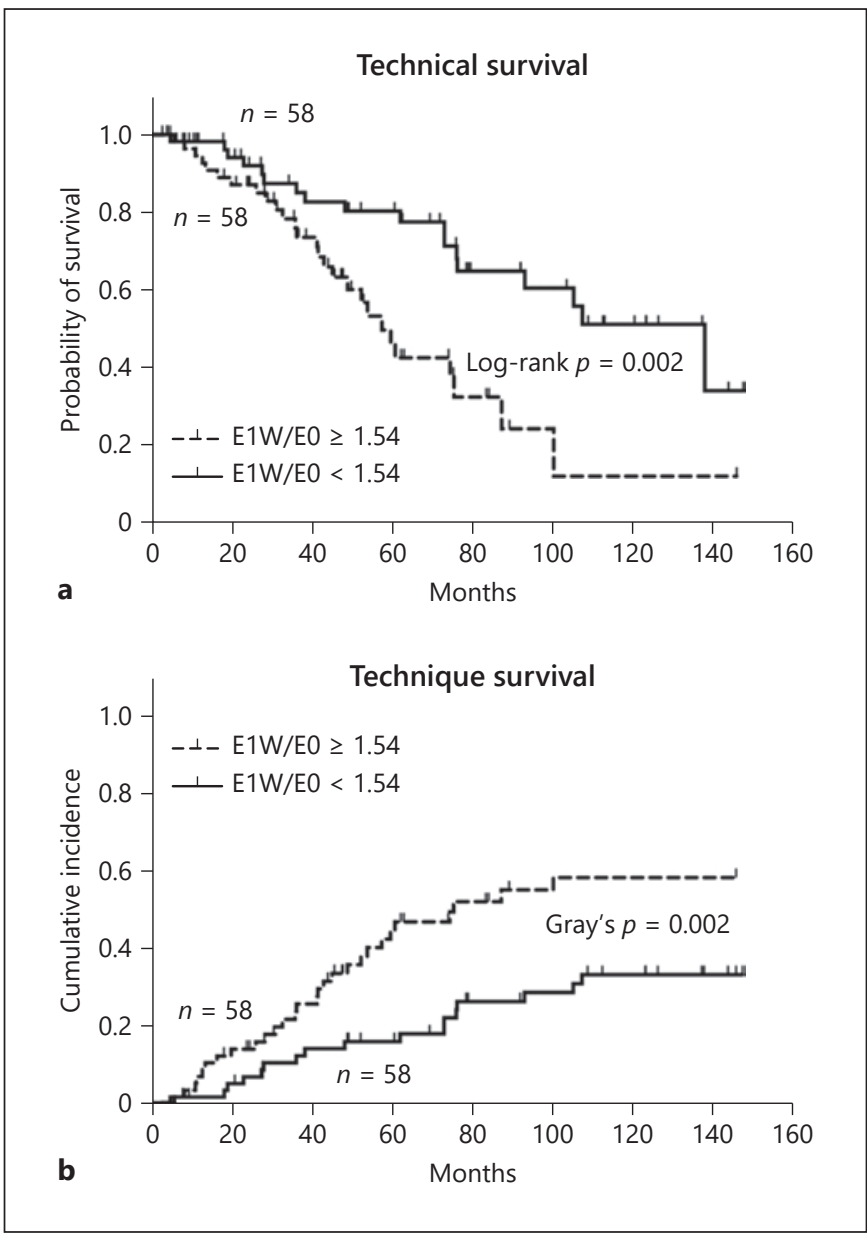

Fig. 3. Kaplan-Meier analysis of the death- and transplant-censored technique survival rate (a) and cumulative incidence curves for technique failure in groups categorized according to the level of E1W/E0 (b). E1W, peripheral blood eosinophil count at 1 week after peritoneal dialysis catheter insertion; E0, peripheral blood eosinophil count before peritoneal dialysis catheter insertion.

0.04 and HR 1.38, $p=0.02$, respectively), whereas male sex exhibited a borderline significant association with a higher risk of technique failure (HR 1.91, $p=0.09$ ). Although E0 was statistically lower in the high E1W/E0 group than in the low E1W/E0 group (Table 1), multivariate analyses included $\mathrm{E} 0$ or $\mathrm{E} 1 \mathrm{~W}$ as independent variables instead of E1W/E0. E0 was not significantly associated with technique failure in both models of regression analysis ( $p=0.46$ for cause-specific hazard model and $p=0.22$ for subdistribution hazard regression model), and $\mathrm{E} 1 \mathrm{~W}$ did not reveal any significant association $(p=$ 0.25 and $p=0.80$, respectively), suggesting that not the absolute value but the elevation of blood EC at the time 
Table 2. Multivariate Cox regression analysis to determine variables associated with technique failure

\begin{tabular}{llll}
\hline Independent variable & HR & $95 \%$ CI & p value \\
& & & \\
\hline Cause-specific hazards & & & \\
Age (per 10 years) & 1.06 & $0.80-1.41$ & 0.66 \\
Sex (male vs. female) & 2.44 & $1.06-5.62$ & 0.04 \\
CCI & 1.47 & $1.15-1.89$ & 0.002 \\
eGFR, mL/min/1.73 m ${ }^{2}$ & 0.92 & $0.75-1.14$ & 0.45 \\
GNRI (per 10) & 0.99 & $0.73-1.35$ & 0.96 \\
E1W/E0 & 1.68 & $1.12-2.53$ & 0.01 \\
\hline Subdistribution hazards & & & \\
Age (per 10 years) & 0.94 & $0.76-1.15$ & 0.55 \\
Sex (male vs. female) & 1.91 & $0.90-4.07$ & 0.09 \\
CCI & 1.38 & $1.06-1.78$ & 0.02 \\
eGFR (mL/min/1.73 m ${ }^{2}$ ) & 0.87 & $0.71-1.07$ & 0.19 \\
GNRI (per 10) & 1.03 & $0.77-1.38$ & 0.85 \\
E1W/E0 & 1.48 & $1.02-2.15$ & 0.04 \\
\hline
\end{tabular}

$\mathrm{HR}$, hazard ratio; $\mathrm{CI}$, confidence interval; eGFR, estimated glomerular filtration rate; GNRI, geriatric nutritional risk index; E1W, peripheral blood eosinophil count at 1 week after peritoneal dialysis catheter insertion; E0, peripheral blood eosinophil count before peritoneal dialysis catheter insertion; CCI, Charlson comorbidity index.

of catheter insertion was the important predictor of technique survival (detailed data not shown). When both E0 and $\mathrm{E} 1 \mathrm{~W}$ were included as independent variables in the cause-specific hazard model, E0 and E1W were associated with lower and higher risks of technique failure (HR 0.68 [per $100 / \mu \mathrm{L}$ ],$p=0.01)$ and $(\mathrm{HR} 1.39$ [per $100 / \mu \mathrm{L}$ ], $p$ $=0.006)$, respectively. However, neither parameter was significantly associated with technique failure in the subdistribution hazard regression model (online suppl. Table 1; see www.karger.com/doi/10.1159/000517349 for all online suppl. material), suggesting that E1W/E0 is the best eosinophil-associated parameter to predict technique survival.

\section{Influence of Eosinophilic Reaction on Peritonitis and $H F$}

Peritonitis was observed in 35 (60\%) and 17 (29\%) patients in the high- and low-E1W/E0 groups, respectively. Peritonitis-free survival time was significantly shorter in the high E1W/E0 group than in the low E1W/E0 group (50.4 vs. 89.8 months, respectively; $p<0.001$ ) (shown in Fig. 4a). In multivariate analysis, standard Cox regression analysis revealed that higher E1W/E0 and higher eGFR were independently associated with the development of

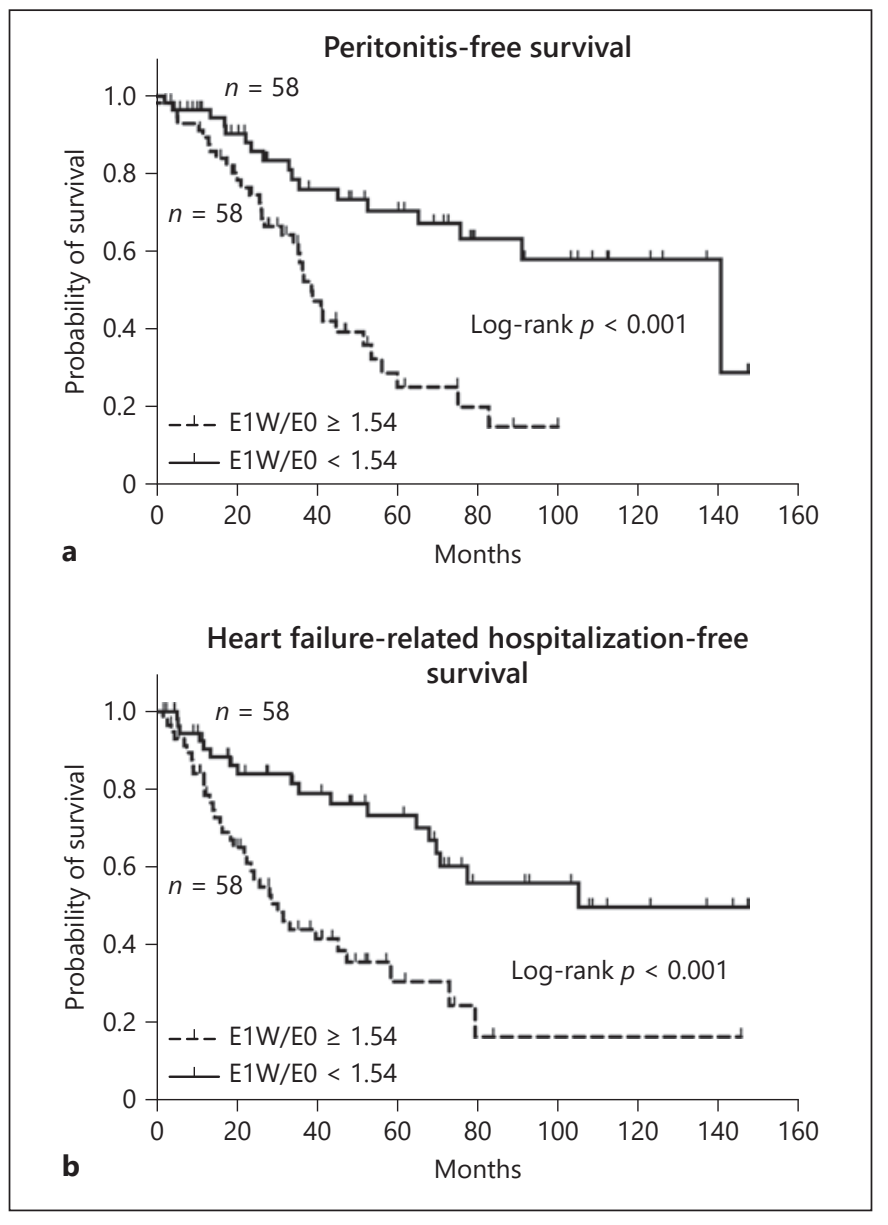

Fig. 4. Kaplan-Meier analysis of the peritonitis-free survival rate (a) and the heart failure-related hospitalization-free survival rate in groups categorized according to median E1W/E0 (b). E1W, peripheral blood eosinophil count at 1 week after peritoneal dialysis catheter insertion; E0, peripheral blood eosinophil count before peritoneal dialysis catheter insertion.

peritonitis (HR 2.19, $p<0.001$ and HR 1.31, $p=0.01$, respectively), whereas higher CCI exhibited a borderline significant association with a higher risk of peritonitis (HR 1.26, $p=0.05$ ) (Table 3). In the multivariate model that included E0 or E1W as independent variables, instead of E1W/E0, E0 was significantly associated with the development of peritonitis (HR $0.71, p=0.006$ ) but E1W was not (detailed data not shown). When including both E0 and E1W as independent variables, E0 and E1W were associated with lower and higher risks of peritonitis (HR $0.45[$ per $100 / \mu \mathrm{L}], p<0.001)$ and (HR $1.65[$ per $100 / \mu \mathrm{L}]$, $p<0.001$, respectively; online suppl. Table 2 ).

The incidence rates of HF-related hospitalization were $60 \%(n=35)$ and $31 \%(n=18)$ in the high- and low-E1W/ 
Table 3. Multivariate Cox regression analysis to determine variables associated with peritonitis and $\mathrm{HF}$

\begin{tabular}{|c|c|c|c|c|c|c|}
\hline \multirow[t]{2}{*}{ Independent variable } & \multicolumn{3}{|c|}{ Peritonitis } & \multicolumn{3}{|c|}{ HF hospitalization } \\
\hline & HR & $95 \%$ CI & $p$ value & HR & $95 \% \mathrm{CI}$ & $p$ value \\
\hline \multicolumn{7}{|l|}{ Cause-specific hazards } \\
\hline Age (per 10 years) & 1.03 & $0.78-1.37$ & 0.81 & 0.87 & $0.66-1.13$ & 0.29 \\
\hline Sex (male vs. female) & 1.30 & $0.64-2.64$ & 0.47 & 2.14 & $0.99-4.61$ & 0.05 \\
\hline CCI & 1.26 & $1.00-1.59$ & 0.05 & 1.29 & $1.02-1.64$ & 0.03 \\
\hline $\mathrm{eGFR}\left(\mathrm{mL} / \mathrm{min} / 1.73 \mathrm{~m}^{2}\right)$ & 1.31 & $1.05-1.63$ & 0.01 & 0.82 & $0.66-1.03$ & 0.08 \\
\hline GNRI (per 10) & 1.14 & $0.88-1.47$ & 0.31 & 1.07 & $0.81-1.40$ & 0.66 \\
\hline E1W/E0 & 2.19 & $1.54-3.10$ & $<0.001$ & 2.15 & $1.51-3.07$ & $<0.001$ \\
\hline
\end{tabular}

HR, hazard ratio; CI, confidence interval; eGFR, estimated glomerular filtration rate; GNRI, geriatric nutritional risk index; E1W, peripheral blood eosinophil count at 1 week after peritoneal dialysis catheter insertion; E0, peripheral blood eosinophil count before peritoneal dialysis catheter insertion; HF, heart failure; CCI, Charlson comorbidity index.

E0 groups, respectively. The HF-related hospitalizationfree survival time was significantly shorter in the highE1W/E0 group than in the low-E1W/E0 group (50.9 vs. 98.3 months, respectively; $p<0.001$ ) (shown in Fig. $4 \mathrm{~b}$ ). Standard Cox regression analysis revealed that higher E1W/E0 and higher CCI were independently associated with a higher risk of HF-related hospitalization (HR 2.15, $p<0.001$ and HR 1.29, $p=0.03$, respectively), whereas male sex tended to be associated with a higher risk of HFrelated hospitalization (HR 2.14, $p=0.05$ ). Additionally, higher eGFR exhibited a borderline significant association with a lower risk of HF-related hospitalization (HR $0.82, p=0.08$ ) (Table 3 ). In the multivariate model that included E0 or E1W as independent variables, instead of E1W/E0, E0 was significantly associated with the development of HF (HR 0.76, $p=0.02$ ), but E1W was not associated with HF (detailed data not shown). When including both E0 and E1W as independent variables, E0 and E1W were associated with lower and higher risks of HF (HR 0.53 [per 100/ $\mu \mathrm{L}$ ], $p<0.001$ ) and (HR 1.44 [per $100 / \mu \mathrm{L}], p<0.001$, respectively; online suppl. Table 2 ).

\section{Discussion}

Compared with hemodialysis, one major issue in $\mathrm{PD}$ is the higher rate of technique failure. Peritonitis and congestive HF, the 2 most frequent causes of technique failure, result in low penetration rates [22]. Therefore, continuous and extensive efforts are ongoing to identify risk factors of technique failure and to develop potential interventions to improve technique survival. Studies identi- fied older age; male sex; diabetes mellitus; presence of comorbidities; lower residual renal function (RRF); lower nutritional status including lower body mass index, albumin, and GNRI; and lower exercise capacity assessed by the incremental shuttle walking or 6-min walking test as factors independently associated with the higher technique failure rate $[17-21,23,24]$. The present study demonstrated that E1W/E0 as readout for eosinophilic reaction at the time of PD catheter insertion was a novel marker that predicted both the higher technique failure rate and higher peritonitis and HF-related hospitalization rates in patients initiating $\mathrm{PD}$. This outcome persisted even after adjusting for known predictive factors including age, sex, CCI, RRF, and GNRI. In contrast to previous reports, RRF was not associated with technique failure in our study. This inconsistency may be due to the use of eGFR, rather than the arithmetic mean of 24-h urea nitrogen and creatinine clearance, which is the gold standard to assess RRF [25-27]. In addition, the rate of RRF decline, which might be a more powerful predictor of technique failure, was not taken into account [27]. Finally, the aggressive use of combined therapy with PD and hemodialysis in Japan may offset the disadvantage of PD in the anuric state with a high risk of fluid overload [28].

The widely reported peripheral blood eosinophilia associated with PD is frequently related to PD catheter insertion [2-11] and icodextrin use [29,30]. Peripheral blood eosinophilia associated with $\mathrm{PD}$, which tends to be mild, has been reported to be associated with peritoneal fluid eosinophilia $[1,2,31]$. The pathophysiology of the increase and activation of eosinophils in the peritoneal fluid is suggested to involve hypersensitivity or foreign 
body reaction to the constituents of the PD system, including sterilants such as ethylene oxide, additives, plasticizers, adhesives, and impurities, as well as silicone rubber catheters and polyester cuffs $[8,11,32]$. Additionally, even innate factors, like the mechanical irritant effect of a large PD fluid volume on peritoneum, saline lavage, and introduction of air in peritoneum, might cause peritoneal fluid eosinophilia [11]. This reaction might be induced and sustained by cytokines, including interleukin 5 , released at the inflammation site, complement activation, and the complements C3a and C5a $[9,33]$. The increase and activation of eosinophils in the blood and $\mathrm{PD}$ fluid have been reported not to cause any adverse effects on peritoneal function or other outcomes in patients on $\mathrm{PD}[2,10,11]$, whereas several studies have demonstrated contradictory findings, including detrimental effects, particularly on the peritoneal membrane, leading to EPS in the worst-case scenario $[12,13,34]$. Although we did not investigate ECs in the PD fluid in this study, the increase in peripheral blood EC persisted for 1 year after $P D$ catheter insertion, which might have been partially exacerbated by the use of the PD fluid including icodextrin and PD systems with bags and tubes in the follow-up period; it remains plausible that the long-term increase and activation of eosinophils in the blood and PD fluid might have had adverse effects in patients on PD. Of note, eosinophils are involved in allergic reactions and trigger immune reactions and evoke effector functions in response to tissue damage $[35,36]$. A recent review suggested that eosinophilic inflammation in peritonea plays a key role in the development of EPS [37]. We first proved that even a modest increase in peripheral blood EC was associated with harmful effects on technique survival in patients initiating PD. The observed eosinophilic reaction was also associated with peritonitis- and HF-related hospitalization-free survival rates; these events might be assumed to be directly related to shortened technique survival. Actually, the proportion of peritonitis as the cause of technique failure tended to be higher in the high E1W/E0 group than in the low E1W/E0 group. In addition, the longitudinal increase in $\mathrm{D} / \mathrm{P} 4$ was greater in the high E1W/E0 group than in the low E1W/E0 group in this study, suggesting that ultrafiltration volume decreased in the high E1W/E0 group, leading to an increased risk of HF. These findings support the hypothesis that long-term increases in EC in the peripheral blood adversely affect peritoneal function. Particularly, long-term increases in EC may increase the susceptibility to peritonitis.

Silicone, the major material in PD catheters, is biocompatible and well-tolerated but has been associated with lo- cal lymphadenopathy and development of autoimmune diseases in rare cases [38]. Many studies have reported the failure of silicone ventriculoperitoneal shunts due to suspected delayed hypersensitivity to silicone [38-41]. One report attributed peripheral blood eosinophilia to allergy against a silicone PD catheter in a patient with a positive patch test using catheter fragments, indicating type IV delayed hypersensitivity to silicone or other PD catheter components [7]. Therefore, desensitization to silicone or other PD catheter components before catheter placement might be effective in reducing eosinophilic reaction at the time of catheter insertion [42], which requires further preclinical and clinical studies.

The present study has several limitations. First, this was an observational cohort study, hindering the complete exclusion of the impact of other potential confounders. Additionally, the study was performed at a single center, and the sample size was small. However, we minimized the effects of covariates by performing multivariate analysis using all known risk factors of technique failure reported in previous studies. The multivariate model had a sufficiently high concordance index for goodness of fit. Third, it was difficult to clarify the products causing eosinophilic reaction at the time of PD catheter insertion. Although we could not specifically exclude the potential effect of a reaction to antibiotics, it is not reasonable to consider that the eosinophilic reaction to antibiotics predicts PD technique survival. Additionally, the potential effect of vancomycin exposure, previously identified as a risk factor for eosinophilic peritonitis, was negligible in this study because only 2 patients were treated with vancomycin perioperatively [31]. Of note, the mean administration period of antibiotics in our study was approximately 5 days, which was longer than the recommendation of the International Society for Peritoneal Dialysis $[43,44]$. Antibiotics were used longer due to the fear of persistent inflammation/infection after surgery involving the insertion of an artificial object. No PD participants developed peritonitis or exit-site/tunnel infection episodes within 2 weeks after catheter insertion, and adverse events associated with the prolonged administration of antibiotics (mostly cefazolin) were rarely detected. However, considering the rationale/recommendation of perioperative administration of antibiotics and the probability of antibiotics causing increased ECs in the peripheral blood, which may lead to the deterioration of outcomes in patients with $\mathrm{PD}$, we actively considered reducing the duration of perioperative antibiotics administration. Finally, EC and biomarkers, including interleukin-6, interleukin-5, and complements, in peritoneal dialysate fluid
Uchiyama/Washida/Kusahana/ Nakayama/Morimoto/Itoh 
were not measured because this observational study was conducted within the health insurance scheme. EC in the peripheral blood can be easily used in routine clinical practice. However, further prospective studies are required to clarify the association of EC and biomarkers in the peritoneal fluid after PD catheter insertion with absolute EC or change in EC in the peripheral blood and PDrelated outcomes.

In conclusion, we demonstrated that eosinophilic reaction at the time of PD catheter insertion was a novel and independent predictor of technique survival, peritonitisfree survival, and HF-related hospitalization-free survival in patients initiating PD. Further studies are warranted to investigate interventions, including desensitization, that can mitigate eosinophilic reaction and improve PD technique survival.

\section{Statement of Ethics}

This study and all its protocols were reviewed and approved by the Ethics Committee of our hospital, and informed consent was obtained from all patients prior to participation.

\section{Conflict of Interest Statement}

All the authors have no conflicts of interest to declare.

\section{Funding Sources}

The authors did not receive any funding.

\section{Author Contributions}

K.U.: designed the study and wrote the initial draft of the manuscript. K.U., N.W., E.K., T.N., T.K., and K.M.: contributed to data collection. K.U. and E.K.: contributed to analysis and interpretation of data and assisted in the preparation of the manuscript. N.W., K.M., and H.I.: supervised the manuscript. All authors have approved the final version of the manuscript and agreed to be accountable for all aspects of the work in ensuring that questions related to the accuracy or integrity of any part of the work are appropriately investigated and resolved.

\section{References}

1 Twardowski ZJ. Peritoneal access: the past, present, and the future. Contrib Nephrol. 2006;150:195-201.

2 Humayun HM, Ing TS, Daugirdas JT, Gandhi VC, Popli S, Robinson JA, et al. Peritoneal fluid eosinophilia in patients undergoing maintenance peritoneal dialysis. Arch Intern Med. 1981 Aug;141(9):1172-3.

3 Chandran PK, Humayun HM, Daugirdas JT, Nawab ZM, Gandhi VC, Ing TS. Blood eosinophilia in patients undergoing maintenance peritoneal dialysis. Arch Intern Med. 1985 Jan;145(1):114-6.

4 Chan MK, Chow L, Lam SS, Jones B. Peritoneal eosinophilia in patients on continuous ambulatory peritoneal dialysis: a prospective study. Am J Kidney Dis. 1988 Feb;11(2):1803.

5 Oh SY, Kim H, Kang JM, Lim SH, Park HD, Jung SS, et al. Eosinophilic peritonitis in a patient with continuous ambulatory peritoneal dialysis (CAPD). Korean J Intern Med. 2004 Jun;19(2):121-3.

6 Jo YI, Song JO, Park JH, Lee JH, Shin SK. Idiopathic eosinophilic peritonitis in continuous ambulatory peritoneal dialysis: experience with percutaneous catheter placement. Nephrology. 2007 Oct;12(5):437-40.

7 Patel UO, Fox SR, Moy JN, Korbet SM. Pruritic rash and eosinophilia in a patient receiving peritoneal dialysis. Semin Dial. 2011 May-Jun;24(3):338-40.
8 Cetin N, Sav NM, Ciftci E, Yildiz B. Foreign body reaction to dialysis chatheter and peritoneal fluid eosinophilia in a child on continuous ambulatory peritoneal dialysis. Iran J Kidney Dis. 2017 Jul;11(4):319-21.

9 Shigemoto E, Mizuno M, Suzuki Y, Kobayashi K, Sakata F, Kariya T, et al. Increase of eosinophil in dialysate during induction of peritoneal dialysis. Perit Dial Int. 2019 Jan-Feb; 39(1):90-2.

10 Ejaz AA, Zabaneh RI, Ridlon T, Durkin W, Siddiqui T. Effect of chronic peritoneal fluid eosinophilia on peritoneal membrane function. J Pak Med Assoc. 1998 Aug;48(8):233-4.

11 Ejaz AA, Fitzpatrick PM, Durkin AJ, Wasiluk A, Haley WE, Goalen MJ, et al. Pathophysiology of peritoneal fluid eosinophilia in peritoneal dialysis patients. Nephron. 1999 Feb; 81(2):125-30.

12 Nakamura Y, Okada H, Yasui A, Koh T, Yamane T. Sclerosing encapsulating peritonitis associated with recurrent eosinophilic peritonitis. Nephrol Dial Transplant. 1999 Mar; 14(3):768-70.

13 Yuzawa Y, Ito Y, Mizuno M, Sawai A, Matsuo S. Pathological changes in chronic eosinophilic peritonitis in peritoneal dialysis patient. NDT Plus. 2010 Aug;3(4):372-5.

14 Kang SH, Cho KH, Park JW, Yoon KW, Do JY. Geriatric nutritional risk index as a prognostic factor in peritoneal dialysis patients. Perit Dial Int. 2013 Jul-Aug;33(4):405-10.
15 Figueiredo A, Goh BL, Jenkins S, Johnson DW, Mactier R, Ramalakshmi S, et al. Clinical practice guidelines for peritoneal access. Perit Dial Int. 2010 Jul-Aug;30(4):424-9.

16 Nakayama M, Miyazaki M, Honda K, Kasai K, Tomo T, Nakamoto H, et al. Encapsulating peritoneal sclerosis in the era of a multi-disciplinary approach based on biocompatible solutions: the NEXT-PD study. Perit Dial Int. 2014 Nov-Dec;34(7):766-74.

17 Kolesnyk I, Dekker FW, Boeschoten EW, Krediet RT. Time-dependent reasons for peritoneal dialysis technique failure and mortality. Perit Dial Int. 2010 Mar-Apr;30(2): 170-7.

18 Chidambaram M, Bargman JM, Quinn RR, Austin PC, Hux JE, Laupacis A. Patient and physician predictors of peritoneal dialysis technique failure: a population based, retrospective cohort study. Perit Dial Int. 2011 Sep-Oct;31(5):565-73.

19 See EJ, Johnson DW, Hawley CM, Pascoe EM, Badve SV, Boudville N, et al. Risk predictors and causes of technique failure within the first year of peritoneal dialysis: an Australia and New Zealand dialysis and transplant registry (ANZDATA) Study. Am J Kidney Dis. 2018 Aug;72(2):188-97. 
20 Boudville N, Ullah S, Clayton P, Sud K, Borlace $\mathrm{M}, \mathrm{Badve} \mathrm{SV}$, et al. Differences in peritoneal dialysis technique survival between patients treated with peritoneal dialysis systems from different companies. Nephrol Dial Transplant. 2019 Jun;34(6): 1035-44.

21 Nakayama T, Uchiyama K, Washida N, Morimoto K, Muraoka K, Adachi K, et al. Exercise parameters predict technique survival in patients on peritoneal dialysis. Blood Purif. 2020 Oct. 1-10, in press.

22 Li PK, Chow KM, Van de Luijtgaarden MW, Johnson DW, Jager KJ, Mehrotra R, et al. Changes in the worldwide epidemiology of peritoneal dialysis. Nat Rev Nephrol. 2017 Feb;13(2):90-103.

23 Chen HL, Tarng DC, Huang LH. Risk factors associated with outcomes of peritoneal dialysis in Taiwan: an analysis using a competing risk model. Medicine. 2019 Feb;98(6):e14385.

24 Shi Y, Zheng D, Zhang L, Yu Z, Yan H, Ni Z, et al. Six-minute walk test predicts all-cause mortality and technique failure in ambulatory peritoneal dialysis patients. Nephrology. 2017 Feb;22(2):118-24.

25 van Olden RW, Krediet RT, Struijk DG, Arisz L. Measurement of residual renal function in patients treated with continuous ambulatory peritoneal dialysis. J Am Soc Nephrol. 1996 May;7(5):745-50.

26 Bargman JM, Thorpe KE, Churchill DN; CANUSA Peritoneal Dialysis Study Group. Relative contribution of residual renal function and peritoneal clearance to adequacy of dialysis: a reanalysis of the CANUSA study. J Am Soc Nephrol. 2001 Oct;12(10):2158-62.

27 Liao CT, Chen YM, Shiao CC, Hu FC, Huang JW, Kao TW, et al. Rate of decline of residual renal function is associated with all-cause mortality and technique failure in patients on long-term peritoneal dialysis. Nephrol Dial Transplant. 2009 Sep;24(9):2909-14.
28 Tanaka M, Ishibashi Y, Hamasaki Y, Kamijo Y, Idei M, Nishi T, et al. Bioimpedance spectroscopy-based fluid status in combined dialysis compared with hemodialysis and peritoneal dialysis: a cross-sectional study. Ther Apher Dial. 2020 Aug;24(4):373-9.

29 MacGinley R, Cooney K, Alexander G, Cohen S, Goldsmith DJ. Relapsing culture-negative peritonitis in peritoneal dialysis patients exposed to icodextrin solution. Am J Kidney Dis. 2002 Nov; 40(5):1030-5.

30 Touré F, Lavaud S, Mohajer M, Lavaud F, Canivet E, Nguyen $P$, et al. Icodextrin-induced peritonitis: study of five cases and comparison with bacterial peritonitis. Kidney Int. 2004 Feb;65(2):654-60.

31 Gauckler P, Shin JI, Mayer G, Kronbichler A. Eosinophilia and kidney disease: more than just an incidental finding? J Clin Med. 2018 Dec;7(12):529.

32 Curtis J, Klykken P. Comments on a case report of a Tenckhoff catheter allergy. Semin Dial. 2011 Nov-Dec;24(6):686-7.

33 DiScipio RG, Schraufstatter IU. The role of the complement anaphylatoxins in the recruitment of eosinophils. Int Immunopharmacol. 2007 Dec;7(14):1909-23.

34 Ikee R, Oka M, Maesato K, Mano T, Moriya $\mathrm{H}$, Ohtake T, et al. Eosinophilic peritonitis and ultrafiltration failure on initiation of CAPD. Perit Dial Int. 2008 Mar-Apr;28(2): 197-9.

35 Kobayashi T, Kouzaki H, Kita H. Human eosinophils recognize endogenous danger signal crystalline uric acid and produce proinflammatory cytokines mediated by autocrine ATP. J Immunol. 2010 Jun;184(11):6350-8.
36 Kobayashi T, Soma T, Noguchi T, Nakagome $\mathrm{K}$, Nakamoto H, Kita H, et al. ATP drives eosinophil effector responses through P2 purinergic receptors. Allergol Int. 2015 Sep; 64(Suppl):S30-6.0

37 Kobayashi T, Noguchi T, Saito K, Shirasaki F, Kita H, Nagata M, et al. Eosinophilic inflammation in peritoneal fibrosis patients undergoing peritoneal dialysis. Contrib Nephrol. 2018;196:1-4.

38 Kurin M, Lee K, Gardner P, Fajt M, Umapathy C, Fasanella K. Clinical peritonitis from allergy to silicone ventriculoperitoneal shunt. Clin J Gastroenterol. 2017 Jun;10(3):229-31.

39 Goldblum RM, Pelley RP, O’Donell AA, Pyron D, Heggers JP. Antibodies to silicone elastomers and reactions to ventriculoperitoneal shunts. Lancet. 1992 Aug;340(8818):510-3.

40 Brownlee JD, Brodkey JS, Schaefer IK. Colonic perforation by ventriculoperitoneal shunt tubing: a case of suspected silicone allergy. Surg Neurol. 1998 Jan;49(1):21-4.

41 Hussain NS, Wang PP, James C, Carson BS, Avellino AM. Distal ventriculoperitoneal shunt failure caused by silicone allergy. Case report. J Neurosurg. 2005 Mar;102(3):536-9.

42 Scherer K, Brockow K, Aberer W, Gooi JH, Demoly P, Romano A, et al. Desensitization in delayed drug hypersensitivity reactions: an EAACI position paper of the Drug Allergy Interest Group. Allergy. 2013 Jul;68(7):844-52.

43 Li PK, Szeto CC, Piraino B, de Arteaga J, Fan $S$, Figueiredo AE, et al. ISPD peritonitis recommendations: 2016 update on prevention and treatment. Perit Dial Int. 2016 Sep;36(5): 481-508.

44 Szeto CC, Li PK, Johnson DW, Bernardini J, Dong J, Figueiredo AE, et al. ISPD catheterrelated infection recommendations: 2017 update. Perit Dial Int. 2017 Mar-Apr;37(2):14154. 